\title{
Full-Commanding a Network: the Dictator
}

\author{
Clara Grácio ${ }^{1}$, Sara Fernandes ${ }^{2}$, and Luís Mário Lopes ${ }^{3}$ \\ 1 Department of Mathematics, Universidade de Évora and CIMA-UE, \\ Rua Romão Ramalho, 59, 7000-671 Évora,Portugal \\ mgracio@uevora.pt \\ 2 Department of Mathematics, Universidade de Évora and CIMA-UE, \\ Rua Romão Ramalho, 59, 7000-671 Évora,Portugal \\ saf@uevora.pt \\ 3 Mathematics Unit, ADM, Instituto Superior de Engenharia de Lisboa, \\ Rua Conselheiro Emídio Navarro, 1, 1949-014 Lisboa, Portugal \\ luismariolopes@gmail.com
}

\begin{abstract}
A network of chaotic dynamical systems may synchronize. For some networks there is the possibility that, coupling a new node to the network, the synchronization will be commanded by that new node. That possibility depends on the network and on the way the new node is coupled to the network. We consider a coupling that can provide what we call a full-commanding and we define the corresponding full-commandwindow. The limit situations corresponding to a completely connected network and to a completely disconnected one provide us some understanding about what makes a network more receptive or more resistant to commanding.
\end{abstract}

Keywords: Chaotic Dynamical Systems, Complete Synchronization, Command, Full-Command Windows, Dictator

\section{Introduction}

The synchronization of coupled chaotic dynamical systems is largely studied in literature [1], [2], [3], [4], [5]. In section 2, we consider a network of identical one-dimensional chaotic discrete dynamical systems connected to each other in a linear way and we obtain conditions for the synchronization to take place. The possibility of commanding the synchronized behavior is obviously a relevant subject. We analyse the possibility to achive that by adding a new node in a full-commanding way, and we call this new node the dictator. In section 3 , we analyse the conditions that a network needs to satisfy to be full-commandable. Even if a network is full-commandable, not all the values of the coupling strength constant that couples the dictator to the other nodes of the network allow the dictator to command the network. Those which do so define the full-commandwindow. In section 3, we also obtain that window and particularize it for a completely connected network and for a completely disconnected one, getting some conclusions about what may provide more resistance or more receptiveness to the full-commanding by a dictator. 


\section{Complete synchronization in a network}

We consider a network of $N$ one-dimensional chaotic discrete dynamical systems, $x_{i}(i=1, \ldots, N)$, with the same dynamic defined by the map $f$, described by

$$
x_{i}(t+1)=f\left(x_{i}(t)\right)+\sum_{\substack{j=1 \\ j \neq i}}^{N} a_{i j} \cdot\left[f\left(x_{j}(t)\right)-f\left(x_{i}(t)\right)\right]
$$

or, equivalently, by

$$
x_{i}(t+1)=\left(1-\sum_{\substack{j=1 \\ j \neq i}}^{N} a_{i j}\right) \cdot f\left(x_{i}(t)\right)+\sum_{\substack{j=1 \\ j \neq i}}^{N} a_{i j} \cdot f\left(x_{j}(t)\right)
$$

where $a_{i j} \in \mathbb{R}_{0}^{+}(i, j=1, \ldots, N)$ are the coupling constants.

Defining $\vec{x}(t)=\left[x_{1}(t) x_{2}(t) \ldots x_{N}(t)\right]^{T}$ and $\vec{f}(\vec{x}(t))=\left[f\left(x_{1}(t)\right) f\left(x_{2}(t)\right) \ldots f\left(x_{N}(t)\right)\right]^{T}$, $a_{i i}=-\sum_{\substack{j=1 \\ j \neq i}}^{N} a_{i j}$ and $A=\left[a_{i j}\right]$, these equations may be written as

$$
\vec{x}(t+1)=\left(I_{N}+A\right) \cdot \vec{f}(\vec{x}(t))
$$

and we call $A$ the coupling matrix.

We note that $\lambda=0$ is an eigenvalue of $A$ (in fact, $A \cdot \overrightarrow{1}=\overrightarrow{0}=0 \cdot \overrightarrow{1}$, with $\left.\overrightarrow{1}=[11 \ldots 1]^{T}\right)$. Further, network (1) admits a completely synchronized solution (see definition hereafter), namely $\vec{x}(t)=x_{1}(t) \cdot \overrightarrow{1}$ is a solution of (1), for any function $x_{1}(t)$ that satisfies $x_{1}(t+1)=f\left(x_{1}(t)\right)$.

Definition 1. We say that the coupled system (1) admits a completely synchronized solution if there is a synchronized function $s(t)$ such that $\left(x_{1}(t), x_{2}(t)\right)=$ $(s(t), s(t))$ is a solution of (1).

The following proposition (a variant of similar results already presented [6], [7]) provides conditions for the existence of an exponentially stable completely synchronized solution.

Proposition 1. Considering the dynamical network (1) with $A$ a diagonalizable matrix such that $\lambda_{1}=0$ is an eigenvalue of multiplicity 1 , if all the other eigenvalues $\lambda_{i}(i=2, \ldots, N)$ are such that $\left|1+\lambda_{i}\right|<e^{-h}$, where $h$ is the Lyapunov exponent of the nodes, then the completely synchronized solution $\vec{x}(t)=x_{1}(t) \cdot \overrightarrow{1}$, with $x_{1}(t)$ satisfying $x_{1}(t+1)=f\left(x_{1}(t)\right)$, is exponentially stable. If $\left|1+\lambda_{i}\right|>e^{-h}$ for a non-zero eigenvalue $\lambda_{i}$, then there is not an exponentially stable completely synchronized solution. 
Proof. Considering $\vec{x}(t)=x_{1}(t) \cdot \overrightarrow{1}+\vec{u}(t)$, the linearization of (1) around $\vec{x}(t)=x_{1}(t) \cdot \overrightarrow{1}$ provides

$$
\begin{aligned}
& x_{1}(t+1) \cdot \overrightarrow{1}+\vec{u}(t+1)= \\
& \quad=\left(I_{N}+A\right) \cdot\left[f\left(x_{1}(t)\right) \overrightarrow{1}+f^{\prime}\left(x_{1}(t)\right) \cdot \vec{u}(t)\right]
\end{aligned}
$$

and, since $A \cdot \overrightarrow{1}=0 \cdot \overrightarrow{1}$ and $x_{1}(t+1)=f\left(x_{1}(t)\right)$, we obtain

$$
\vec{u}(t+1)=\left(I_{N}+A\right) \cdot f^{\prime}\left(x_{1}(t)\right) \cdot \vec{u}(t)
$$

Considering $D=\operatorname{diag}\left(0, \lambda_{2}, \lambda_{3}, \ldots, \lambda_{N}\right)$, a diagonal matrix similar to $A$, and $\vec{v}(t)=S \cdot \vec{u}(t)$, with $S$ such that $D=S A S^{-1}$, the previous equation is equivalent to

$$
\begin{gathered}
\vec{v}(t+1)=\left(I_{N}+D\right) \cdot f^{\prime}\left(x_{1}(t)\right) \cdot \vec{v}(t) \\
\Leftrightarrow \\
\left\{\begin{array}{l}
v_{1}(t+1)=f^{\prime}\left(x_{1}(t)\right) \cdot v_{1}(t) \\
v_{i}(t+1)=\left(1+\lambda_{i}\right) \cdot f^{\prime}\left(x_{1}(t)\right) \cdot v_{i}(t), \quad i=2, \ldots, N
\end{array}\right.
\end{gathered}
$$

Then, for $i=2, \ldots, N$,

$$
v_{i}(t+T)=\left(1+\lambda_{i}\right)^{T} \cdot \prod_{\tau=t}^{t+T} f^{\prime}\left(x_{1}(\tau)\right) \cdot v_{i}(t),
$$

and since the Lyapunov exponent $h$ of $x_{1}(t+1)=f\left(x_{1}(t)\right)$ is $h=\lim _{T \rightarrow+\infty} \frac{\sum_{\tau=t}^{t+T} \ln \left|f^{\prime}\left(x_{1}(\tau)\right)\right|}{T}$, we obtain

$$
\begin{aligned}
& \lim _{T \rightarrow+\infty}\left|v_{i}(t+T)\right|= \\
& \quad=\lim _{T \rightarrow+\infty}\left|\left(1+\lambda_{i}\right)^{T}\right| \cdot e^{\ln \prod_{\tau=t}^{t+T}\left|f^{\prime}\left(x_{1}(\tau)\right)\right|} \cdot\left|v_{i}(t)\right|= \\
& \quad=\lim _{T \rightarrow+\infty}\left(\left|1+\lambda_{i}\right| e^{h}\right)^{T} \cdot\left|v_{i}(t)\right|
\end{aligned}
$$

If $\left|1+\lambda_{i}\right| e^{h}<1$ or, equivalently, $\left|1+\lambda_{i}\right|<e^{-h}$ for $i=2, \ldots, N$, then the completely synchronized solution $\vec{x}(t)=x_{1}(t) \cdot \overrightarrow{1}$ is exponentially stable.

If there is a $i \in\{2, \ldots, N\}$ such that $\left|1+\lambda_{i}\right| e^{h}>1$ or, equivalently, $\left|1+\lambda_{i}\right|>$ $e^{-h}$, then there is not an exponentially stable completely synchronized solution.

Now, we introduce another node $y$ - the dictator - and we want to analyse how it can command the network and how the network can resist to its command. The analysis done in [8] regarding what was called there the Commanded Linear Coupled System, sugests that we make a one-way connection of the new node $y$ to all the other nodes. We will consider a full-commanding: one in which the dictator is connected to all other nodes with the same commanding coupling strength constant $\epsilon \in \mathbb{R}_{0}^{+}$. 


\section{Full-commanding}

Using a new node $y$ to full-command the network (1) originates the following new network:

$$
\left\{\begin{array}{l}
x_{i}(t+1)=f\left(x_{i}(t)\right)+\sum_{\substack{j=1 \\
j \neq i}}^{N} a_{i j} \cdot\left[f\left(x_{j}(t)\right)-f\left(x_{i}(t)\right)\right]+\epsilon \cdot\left[f\left(y(t)-f\left(x_{i}(t)\right)\right]\right. \\
y(t+1)=f(y(t))
\end{array}\right.
$$

with $i=1, \ldots, N$, or, equivalently,

$$
\left\{\begin{array}{l}
x_{i}(t+1)=\left(1-\sum_{\substack{j=1 \\
j \neq i}}^{N} a_{i j}-\epsilon\right) \cdot f\left(x_{i}(t)\right)+\sum_{\substack{j=1 \\
j \neq i}}^{N} a_{i j} \cdot f\left(x_{j}(t)\right)+\epsilon \cdot f(y(t)) \\
y(t+1)=f(y(t))
\end{array}\right.
$$

Defining $\overrightarrow{x_{0}}(t)=\left[x_{1}(t) x_{2}(t) \ldots x_{N}(t) y(t)\right]^{T}$ and $A_{0}=\left[\begin{array}{cc}A-\epsilon I_{N} & \epsilon \overrightarrow{1} \\ \overrightarrow{0}^{T} & 0\end{array}\right]$, these equations may be written as

$$
\overrightarrow{x_{0}}(t+1)=\left(I_{N+1}+A_{0}\right) \cdot \vec{f}\left(\overrightarrow{x_{0}}(t)\right)
$$

Proposition 2. If $\left|\epsilon-\left(1+\lambda_{i}\right)\right|<e^{-h}$, for all the eigenvalues $\lambda_{i}$ of the diagonalizable coupling matrix $A$ of the network (1), then the dictator y commands the network, i.e. $\overrightarrow{x_{0}}(t)=y(t) \cdot \overrightarrow{1}$ is an exponentially stable solution of (2). If $\left|\epsilon-\left(1+\lambda_{i}\right)\right|>e^{-h}$ for an eigenvalue $\lambda_{i}$, then $\overrightarrow{x_{0}}(t)=y(t) \cdot \overrightarrow{1}$ is not an exponentially stable solution of (2), i.e. the dictator is not able to command the network in a full-commanding way.

Proof. The eigenvalues of $A_{0}$ are $\lambda=0$ and $\lambda=\lambda_{i}-\epsilon$. Since the sum of the entries in each row of $A_{0}$ is equal to zero and the off-diagonal elements are nonnegative, lemma 2 of [9] assures that $\operatorname{Re}\left(\lambda_{i}\right) \leq 0$ for all eigenvalues $\lambda_{i}$ of $A$. Further $\epsilon \in \mathbb{R}_{0}^{+}$. Then $A_{0}$ has just one zero eigenvalue and Proposition 1 determines that if $\left|1+\lambda_{i}-\epsilon\right|<e^{-h}$ or, equivalently, $\left|\epsilon-\left(1+\lambda_{i}\right)\right|<e^{-h}$, then the synchronized solutions $\overrightarrow{x_{0}}(t)=x_{1}(t) \cdot \overrightarrow{1}$, with $x_{1}(t)$ satisfying $x_{1}(t+1)=$ $f\left(x_{1}(t)\right)$, are exponentially stable. Since $y$ is a free node, i.e. since $y$ satisfies $y(t+1)=f(y(t))$, then $\overrightarrow{x_{0}}(t)=y(t) \cdot \overrightarrow{1}$ is an exponentially stable solution of (2).

Proposition 1 also determines that if $\left|\epsilon-\left(1+\lambda_{i}\right)\right|>e^{-h}$ for at least one of the eigenvalues $\lambda_{i}$ of $A$, then $\overrightarrow{x_{0}}(t)=y(t) \cdot \overrightarrow{1}$ is not an exponentially stable solution of (2). 
This lead us to define

Definition 2. We define full-command-window (FCW) of the network (1) as the open set of values of the commanding coupling constant $\epsilon$ for which the synchronized solution $\overrightarrow{x_{0}}(t)=y(t) \cdot \overrightarrow{1}$ is an exponentially stable solution of (2). If $F C W \neq \varnothing$, we say that network (1) is full-commandable.

Proposition 2 determines that

$$
F C W=\bigcap_{i=1}^{N}\left\{\epsilon \in \mathbb{R}_{0}^{+}:\left|\epsilon-\left(1+\lambda_{i}\right)\right|<e^{-h}\right\}
$$

and we note that there are networks that are not full-commandable, as is established in the following proposition.

Proposition 3. If a diagonalizable coupling matrix A has two eigenvalues that are distant from each other more than $2 e^{-h}$, then the network (1) with the coupling matrix $A$ is not full-commandable. That is also true if $A$ has an eigenvalue $\lambda$ such that $\operatorname{Im}(\lambda)>e^{-h}$.

Proof. We name $\lambda_{1}$ and $\lambda_{2}$ the two eigenvalues of $A$ such that $\left|\lambda_{1}-\lambda_{2}\right|>$ $2 e^{-h}$. Let us suppose that $A$ is full-commandable. Then, Proposition 2 assures that $\left|\epsilon-\left(1+\lambda_{1}\right)\right| \leq e^{-h}$ and $\left|\epsilon-\left(1+\lambda_{2}\right)\right| \leq e^{-h}$, providing $\left|\lambda_{1}-\lambda_{2}\right|=$ $\left|\left[\epsilon-\left(1+\lambda_{2}\right)\right]-\left[\epsilon-\left(1+\lambda_{1}\right)\right]\right| \leq\left|\epsilon-\left(1+\lambda_{1}\right)\right|+\left|\epsilon-\left(1+\lambda_{1}\right)\right| \leq e^{-h}+e^{-h}=$ $2 e^{-h}$. This contradicts our assumption and, so, we conclude that $A$ is not fullcommandable.

If there is a $\lambda$ such that $\operatorname{Im}(\lambda)>e^{-h}$ then

$$
|\epsilon-(1+\lambda)|=\sqrt{(\epsilon-1-\operatorname{Re}(\lambda))^{2}+(\operatorname{Im}(\lambda))^{2}}>\operatorname{Im}(\lambda)>e^{-h}
$$

and so the network is not full-commandable.

There are situations for which we can obtain a simpler description of $F C W$.

Proposition 4. Considering a network (1) such that $A$ is diagonalizable, all its eigenvalues are real and $\lambda_{N}$ is the smallest one, then the network is fullcommandable if $\lambda_{N}>-2 e^{-h}$ and $F C W$ reduces to $] 1-e^{-h}, 1+\lambda_{N}+e^{-h}[$.

Proof. Since $A$ is diagonalizable, we can use Proposition 2. If all the eigenvalues of $A$ are real, then $\left.F C W=\bigcap_{i=1}^{N}\right] 1+\lambda_{i}-e^{-h}, 1+\lambda_{i}+e^{-h}\left[\right.$. If $\lambda_{1}$ and $\lambda_{N}$ are the largest and smallest eigenvalues of $A$, respectively, the intersection that defines the $F C W$ will be nonempty if and only if $1+\lambda_{N}+e^{-h}>$ 
$1+\lambda_{1}-e^{-h}$ or, equivalently, $\lambda_{N}>\lambda_{1}-2 e^{-h}$ and, in that situation $F C W=$ ] $1+\lambda_{1}-e^{-h}, 1+\lambda_{N}+e^{-h}$ [. Since the sum of the entries in each row of $A$ is equal to zero and the off-diagonal elements are nonnegative, lemma 2 of [9] determines that $\lambda_{i} \leq 0$. Further, zero is an eigenvalue of $A$ associated to the eigenvector $\overrightarrow{1}$. So, $\lambda_{1}=0$ and we conclude that the network (1) is fullcommandable if $\lambda_{N}>-2 e^{-h}$ and $\left.F C W=\right] 1-e^{-h}, 1+\lambda_{N}+e^{-h}[$.

It is worthwhile to emphasize that for a full-commandable network in the conditions of the previous Proposition, the smallest commanding coupling constant is $\epsilon=1-e^{-h}$, a value that does not depend on the structure of the network, it only depends on the dynamic of the nodes.

Since a symmetric matrix is diagonalizable and all its eigenvalues are real, we can use Proposition 5 for a symmetric network (1), i.e for a network (1) corresponding to a symmetric coupling matrix. We consider two limit situations in what respects to connectedness:

Proposition 5. A completely disconnected network, i.e. a network (1) with a zero matrix $A$, is full-commandable and its full-command-window is $] 1-e^{-h}, 1+e^{-h}[$.

Proof. All the eigenvalues of a zero matrix are zero. So, Proposition 5 determines that the network is full-commandable (since $0>-2 e^{-h}$ ) and $F C W=$ ] $1-e^{-h}, 1+e^{-h}[$.

Proposition 6. A completely connected network, i.e. a network (1) with

$$
A=c \cdot\left[\begin{array}{ccccc}
-(N-1) & 1 & 1 & \ldots & 1 \\
1 & -(N-1) & 1 & \ldots & 1 \\
1 & 1 & -(N-1) & \ldots & 1 \\
\ldots & \ldots & \ldots & \ldots & \ldots \\
1 & 1 & 1 & \ldots & -(N-1)
\end{array}\right]
$$

where $c$ is the global coupling strength, is full-commandable if $N c<2 e^{-h}$ and its full-command-window is $] 1-e^{-h}, 1-N c+e^{-h}[$.

Proof. Considering $\operatorname{det}\left(\frac{1}{c} A-\lambda I_{N}\right)$, if we substitute the first row $\left(R_{1}\right)$ by the sum of all the rows $\left(\sum_{i=1}^{N} R_{i}\right)$, and all the remaining rows $\left(R_{j}\right.$, with $\left.j=2, \ldots, N\right)$ by $R_{j}+\frac{1}{\lambda} \sum_{i=1}^{N} R_{i}$, we obtain 


$$
\begin{aligned}
\operatorname{det} & \left(\frac{1}{c} A-\lambda I_{N}\right)= \\
= & \operatorname{det}\left[\begin{array}{ccccc}
-\lambda & -\lambda & -\lambda & \ldots & -\lambda \\
0 & -N-\lambda & 0 & \ldots & 0 \\
0 & 0 & -N-\lambda & \ldots & 0 \\
\ldots & \ldots & \ldots & \ldots & \ldots \\
0 & 0 & 0 & \ldots-N-\lambda
\end{array}\right]= \\
= & -\lambda \cdot(-N-\lambda)^{N-1},
\end{aligned}
$$

i.e. $\lambda=0$ and $\lambda=-N$ are the eigenvalues of $\frac{1}{c} A$ of multiplicity 1 and $N-1$, respectively. So, the eigenvalues of $A$ are $\lambda=0$ and $\lambda=-N c$ and Proposition 5 determines that if $N c<2 e^{-h}$ then the completely connected network is full-commandable and that its full-command-window is $F C W=$ ] $1-e^{-h}, 1-N c+e^{-h}[$.

\section{Conclusions}

Some networks (1) may be full-commanded by a dictator. The full-commanding possibility depends on the structure of the network (namely on the eigenvalues of coupling matrix $A$ ) and on the nature of the nodes (namely on its Lyapunov exponent). If the network is symmetric, or if at least all the eigenvalues of $A$ are real, the minimum effort (i.e. the minimum value of the commanding coupling strength constant) that needs to be used in order to the dictator to command the network just depends on the nature of the nodes (namely on its Lyapunov exponent). While a completely disconnected network is always full-commandable, a completely connected one is as more difficult to command, as larger is the network (larger $N$ ) and as stronger are the connections between nodes (larger c).

\section{References}

1. Pikovsky, A., Rosenblum, M., Kurths, J., Synchronization, A Universal Concept in Nonlinear Sciences. Cambridge University Press, Cambridge; 2001.

2. Hasler, M., Maistrenko, Y. L.: An Introduction to the Synchronization of Chaotic Systems: Coupled Skew Tent Maps. IEEE Trans. Circuits Syst. I 1997; vol. 44, no.10, p. 856-66.

3. Sushchik Jr., M. M., Rulkov, N. F., Abarbanel, H. D. I.: Robustness ad Stability of Synchronized Chaos: An Illustrative Model. IEEE Trans. Circuits Syst. I 1997; vol. 44, no.10, p. 867-73.

4. G. Rangarajan, M. Z. Ding, Stability of Synchronized Chaos in Coupled Dynamical Systems. Phys. Lett. A 2002;296:204-9.

5. M. Z. Ding, W. M. Yang, Stability of synchronous chaos and on-off intermittency in coupled map lattices. Phys. Rev. E 1997; 56, no. 4, p. 4009-16.

6. J. Feng, J. Jost and M. Qian, Networks: From Biology to Theory. Springer; 2007.

7. X. Li, G. Chen, Synchronization and Desynchronization of Complex Dynamical Networks: An Engineering Viewpoint. IEEE Trans. Circuits Syst. I 2003; vol. 50, no.11, p. $1381-90$. 
8. Lopes, L., Fernandes, S., Grácio, C.: "Windows of Synchronization" and "Nonchaotic Windows". ESAIM: Proceedings and Surveys 2014;46:161-74.

9. C. W. Wu, L. O. Chua, Synchronization in an array of linearly coupled dynamical systems. IEEE Trans. Circuits Syst. I 1995; vol. 42, p. 430-47. 